\title{
Nonrepetitive sequences on arithmetic progressions
}

\author{
Jarosław Grytczuk * \\ Department of Theoretical Computer Science \\ Jagiellonian University \\ Kraków, Poland \\ Faculty of Mathematics and Information Science \\ Warsaw University of Technology \\ Warszawa, Poland \\ grytczuk@tcs.uj.edu.pl \\ Jakub Kozik ${ }^{\dagger}$ \\ Department of Theoretical Computer Science \\ Jagiellonian University \\ Kraków, Poland \\ jkozik@tcs.uj.edu.pl \\ Marcin Witkowski \\ Faculty of Mathematics and Computer Science \\ Adam Mickiewicz University \\ Poznań, Poland \\ mw@amu . edu .pl
}

Submitted: Apr 14, 2011; Accepted: Sep 18, 2011; Published: Oct 31, 2011

Mathematics Subject Classifications: 68R15, 05D40, 11B25

\begin{abstract}
A sequence $S=s_{1} s_{2} \ldots s_{n}$ is said to be nonrepetitive if no two adjacent blocks of $S$ are identical. In 1906 Thue proved that there exist arbitrarily long nonrepetitive sequences over 3-element set of symbols. We study a generalization of nonrepetitive sequences involving arithmetic progressions. We prove that for every $k \geqslant 1$, there exist arbitrarily long sequences over at most $2 k+10 \sqrt{k}$ symbols whose subsequences, indexed by arithmetic progressions with common differences from the set $\{1,2, \ldots, k\}$, are nonrepetitive. This improves a previous bound of $e^{33} k$ obtained by
\end{abstract}

\footnotetext{
${ }^{*}$ Research of J. Grytczuk is supported by the Polish Ministry of Science and Higher Education grant (MNiSW) (N N206 257035).

${ }^{\dagger}$ Research of J. Kozik is supported by the Polish Ministry of Science and Higher Education grant (MNiSW) (N206 3761 375).
} 
Grytczuk. Our approach is based on a technique introduced recently by Grytczuk Kozik and Micek, which was originally inspired by a constructive proof of the Lovász Local Lemma due to Moser and Tardos. We also discuss some related problems that can be attacked by this method.

\section{Introduction}

For a sequence $S=s_{1} s_{2} \ldots s_{n}$ a repetition of size $h$ is a block (subsequence of consecutive terms) of the form $X X=x_{1} \ldots x_{h} x_{1} \ldots x_{h}$. A sequence is nonrepetitive if it does not contain a repetition of any size $h \geqslant 1$. For example, the sequence 1231312 contains a repetition 3131 of size two, while 123132123 is nonrepetitive.

It is easy to see that the longest nonrepetitive sequence, which can be constructed over a set of two symbols, has length three. In 1906 Thue [18] proved, by a remarkable inductive construction, that there exist arbitrarily long nonrepetitive sequences over just three different symbols (see also [5, 4]). This discovery resulted in many unexpected applications inspiring a stream of research and leading to the emergence of new branches of mathematics with a variety of challenging open problems (see $[1,3,8,11,14]$ ).

A particular variant, proposed in [3], concerns nonrepetitive tilings, i.e., assignments of symbols to lattice points of the plane so that all lines in prescribed directions are nonrepetitive. This idea led Currie and Simpson [7] to consider sequences with a stronger property: all subsequences taken over arithmetic progressions of bounded common differences are nonrepetitive. Let $k \geqslant 1$ be a fixed positive integer and let $S(k)$ be the family of subsequences of $n$-element sequence $S$, of the form $s_{i} s_{i+d} s_{i+2 d} \ldots s_{i+t d}$ with $d \in\{1,2, \ldots, k\}$, $1 \leqslant i \leqslant d-1, t=\lfloor n / d\rfloor$. If every element of $S(k)$ is a nonrepetitive sequence, then $S$ is called nonrepetitive up to $\bmod k$ (see [7]). Let $M(k)$ denote the minimal number of symbols needed to create arbitrarily long sequences nonrepetitive up to mod $k$. Thue's theorem can be rephrased as $M(1)=3$. It is easy to see that $M(k) \geqslant k+2$ for every $k \geqslant 1$, and one may suspect that equality always holds.

Conjecture 1. $M(k)=k+2$ for every $k \geqslant 1$.

This conjecture has been confirmed so far only for $k=2,3$, and 5 (it is not even known for $k=4$ ) by providing Thue type constructions of the desired sequences. However, using the Lovász Local Lemma (see [2]) it was proved in [9] that $M(k) \leqslant e^{33} k$ for any $k$. In this paper we improve the last bound substantially by proving that $M(k) \leqslant 2 k+O(\sqrt{k})$. Our method is inspired by the recent constructive proof of the Lovász Local Lemma due to Moser and Tardos [15]. Just like in the related paper [12] (and in the original nonconstructive approach), we prove the result for a more general version where symbols are chosen from prescribed lists (sets) assigned to the positions in a sequence. The same method applies in the case when $K$ is any $k$-element set of positive integers, and we want to construct an arbitrarily long sequence with no repetitions on arithmetic subsequences with differences from $K$. 


\section{The algorithm}

We present an algorithm that generates consecutive terms of a sequence $S$ by choosing symbols at random (uniformly and independently), and every time a repetition occurs, it erases the longest repeated block and continues from the smallest unassigned position. We always erase the block that contains the last chosen element in order to ensure that after this removal the remaining sequence stays nonrepetitive. In the listing of the algorithms value 0 of some $s_{i}$ means that no symbol is assigned to $s_{i}$. In the beginning each $s_{i}$ equals 0 .

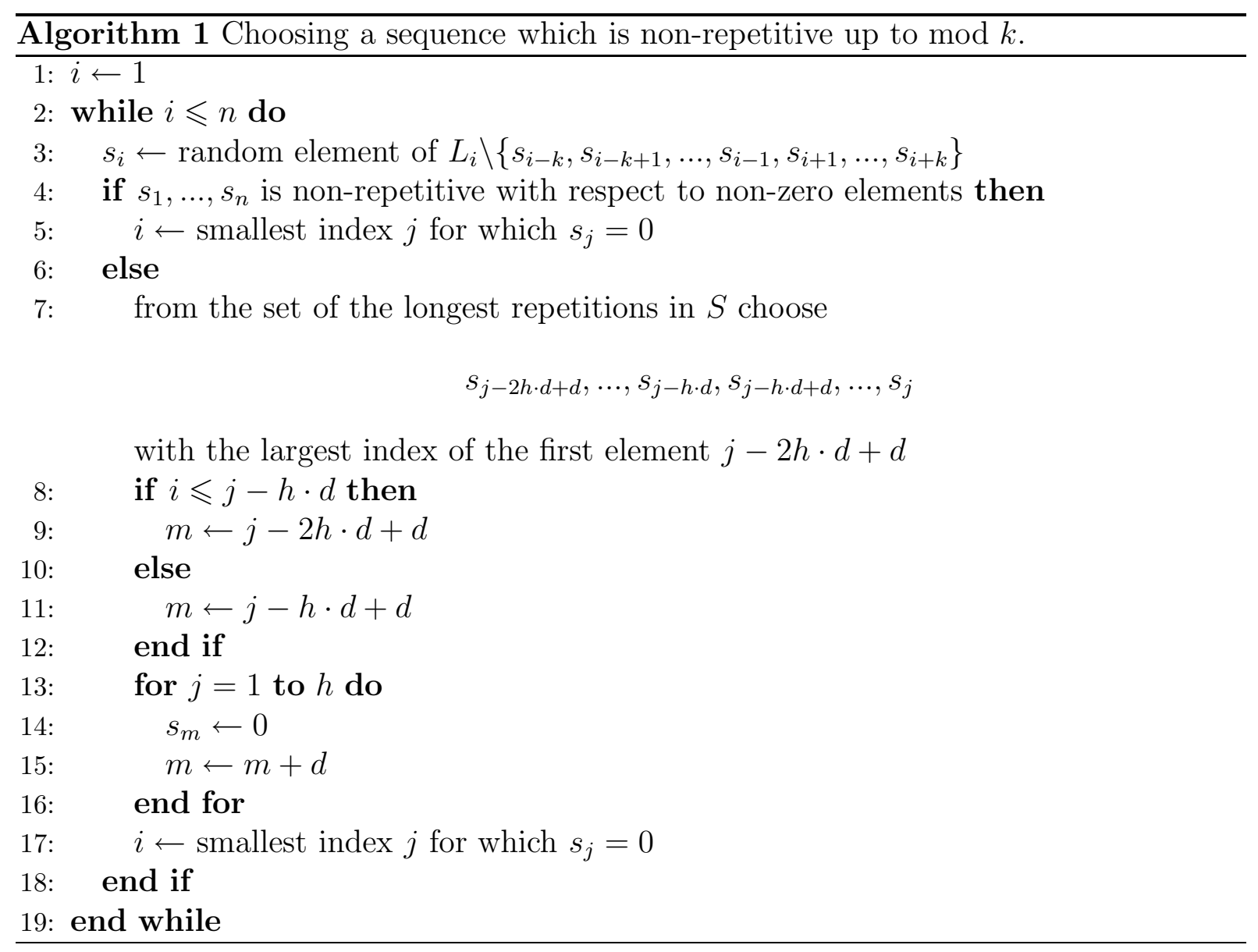

From this point on, whenever we refer to an Algorithm we mean the Algorithm 1. We show that for any given positive integer $n$, and arbitrary lists of symbols $L_{i}$, each of size at least $2 k+10 \sqrt{k}$, the Algorithm computes a sequence of length $n$ which is nonrepetitive up to $\bmod k$. Random elements in line (3) of the Algorithm are chosen independently with uniform distribution. The general idea is to prove that the Algorithm cannot work forever for all possible evaluations of the random experiments. It is easy to see that the Algorithm stops only if a nonrepetitive up to $\bmod k$ sequence is constructed.

Theorem 1. For every positive integer $n$, and for every sequence of sets $L_{1}, \ldots, L_{n}$, each 
of size at least $2 k+10 \sqrt{k}$, there is a sequence $S=s_{1} \ldots s_{n}$ nonrepetitive up to mod $k$ such that $s_{i} \in L_{i}$ for every $i=1,2, \ldots, n$.

Proof. Let us suppose for a contradiction that such sequence does not exist. It means that the Algorithm never stops. We are going to count the possible sequences of random values used in line (3) of the algorithm in two ways.

Let $r_{j}, 1 \leqslant j \leqslant M$, be sequence of values chosen in line (3) in the first $M$ choices of some run of the Algorithm. Each $r_{j}$ can take at least $10 \sqrt{k}$ values. It means that there are at least $10^{M} k^{M / 2}$ such sequences.

The second way of counting involves descriptions of the behaviour of the Algorithm. For every fixed evaluation of the first $M$ random choices we define the following five elements:

- A route $R$ on the upper right quadrant of a $\mathbb{Z} \times \mathbb{Z}$ grid from coordinate $(0,0)$ to coordinate $(2 M, 0)$ on $2 M$ steps with possible moves $(1,1)$ and $(1,-1)$ which never goes below the axis $y=0$.

- A sequence $D$ of numbers between 1 and $k$ corresponding to the peaks on the route $R$, where by a peak we mean a move $(1,1)$ followed immediately by a move $(1,-1)$.

- A sequence $O$ of numbers -1 , or 1 corresponding to the peaks on route $R$.

- A sequence $P$ of integers, one for every peak, whose sum is not greater than $M$.

- A sequence $S$ produced by the Algorithm after $M$ steps.

A pentad $(R, D, O, P, S)$ will be called a $\log$. We encode consecutive steps of the Algorithm into log in the following way:

Each time the algorithm executes line (3) we append a move $(1,1)$ to the route $R$ and for every execution of line $(14)$ we append $(1,-1)$. Notice that in line (14) the algorithm can set zero only to $s_{c}$ which are non-zero. Therefore, the number of down-steps in route $R$ never excess the number of up-steps, and it never goes below axis $y=0$. At the end of computations we add to the route $R$ one down-step for each element of $S$ which is non-zero. This brings us to the point $(2 M, 0)$. Whenever Algorithm executes line (7) we append to the sequence $D$ a difference $d$ of the chosen longest repetition. Next, if $(8)$ is true, then we append 1 to the sequence $O$, otherwise we append -1 . For each execution of the loop (13)-(16), we append to the the sequence $P$, the value $j$ for which $m$ equals $i$ in the loop. Finally, $S$ is the sequence produced by the Algorithm after $M$ executions of line $(3)$.

Claim 1. Every log corresponds to a unique sequence $r_{j}, 1 \leqslant j \leqslant M$ of the first $M$ values chosen in the line (3) in some execution of the Algorithm.

Proof. For a given $\log (R, D, O, P, S)$ we are going to decode $r_{1}, \ldots, r_{M}$. At first we use information from route $R$ and sequences $D$ and $P$ to determine which $s_{i}$ were non-zero at each step of the Algorithm and to find coordinates of elements which were nullified at 
step (14) of the Algorithm. Notice that each operation of setting a non-zero value to some $s_{i}$ corresponds to the up-step $(1,1)$ on the route $R$, while each zeroing of $s_{i}$ corresponds to some down-step $(1,-1)$ in route $R$. We examine the route $R$ from the point $(0,0)$ to the point $(2 M, 0)$. Assume that the first peak occurs after the $j$ th step. Since this is the first time we erase some elements $s_{i}$, we know that $s_{1}, \ldots, s_{j}$ are the only non-zero elements at this point. Now we use information encoded in $D$ and $P$. We look at the number of consecutive down-steps on $R$ (which in this case is equal to $p_{1}$ ) and remember that for this peak we zeroed $s_{j}, s_{j-d_{1}}, s_{j-2 d_{1}}, \ldots, s_{j-\left(p_{1}-1\right) d_{1}}$. Now again each up-step on $R$ denotes setting some value to the zeroed position with the smallest index $i$. Proceeding in that way we know exactly which position was set last, when we reach the next peak. From the number of consecutive down-steps on $R$ we deduce the length of the zeroed repeated block. Value in the sequence $D$ corresponding to the peak denotes the difference of the arithmetic subsequence in which the repetition occurred. Finally corresponding value from the sequence $P$ describes the position of the symbol just set, within the erased repeated blocks. From all this information it is easy to deduce which positions was zeroed as a result of erasing the repetition. We repeat these operations until we get to the end of $R$.

After this preparatory step we are ready to decode $r_{1}, \ldots, r_{M}$. We consider the sequence $R$ in reverse order - from the last point $(2 M, 0)$ to the first $(0,0)$ modifying the final sequence $S$. This time we use information encoded in $S$ and $O$, and the knowledge determined in the preparatory step. As before, each up-step $(1,1)$ on the route $R$ corresponds to some $r_{i}$. For every such up-step we have already determined the indices of elements $r_{i}$ in $S$ in the preparatory analysis. At the beginning, going backward on $R$, there is some number of down-steps corresponding to non-zero elements of $S$ (the elements added at the end of computations). We skip them and move on. Now, each time there is an up-step on $R$, we assign to $r_{j}$ a value from appropriate $s_{i}$ (where $i$ was determined in the preparatory step), and set $s_{i}$ to 0 . In fact, to determine the real outcome of random experiments (i.e. an index of the chosen element on the list of elements available at this step), we must take into account the forbidden symbols from $k$ preceding and $k$ following places on $S$. Every consecutive sequence of $t$ down-steps on $R$ corresponds to the deletion of some repeated block during the execution of the Algorithm. Next we assign to $s_{i}, s_{i+d_{l}}, \ldots, s_{i+(t-1) d_{l}}$ corresponding values $s_{i+o_{l} t d_{l}}, s_{i+d_{l}+o_{l} t d_{l}}, \ldots, s_{i+(t-1) d_{l}+o_{l} t d_{l}}$ (where $s_{i}$ is the first element of the erased repeated block determined in the preparatory step). These are exactly the values from the repetitions erased at step (17) of the Algorithm.

We showed that each sequence of randomly chosen values during the execution of the Algorithm corresponds to some log, and that this mapping is injective. As a consequence the number of different $\operatorname{logs}_{\mathrm{s}}$ is always greater than or equal to the number of feasible sequences $r_{1}, \ldots, r_{M}$. Let $L$ be the size of the set of all possible logs. To calculate $L$ we have to determine the number of different structures for each element in a log. The number of all possible routes on the upper right quadrant of a grid of length $2 M$ with possible moves $(1,1)$ and $(1,-1)$ is well known to be the $M$ th Catalan number $C_{M}$. Since in every choice in line (3) the elements occurring within distance $k$ are excluded, the Algorithm cannot produce repetition that contains less than 4 elements. It means that 
the subsequence $(1,1),(1,-1),(1,1)$ can not occur in the route $R$. Therefore the number of peaks within $R$ cannot exceed $M / 2$. Thus there can be at most $k^{M / 2}$ possible sequences $D$. Respectively, there are at most $2^{M / 2}$ possible evaluations for sequence $O$.

The sequence $S$ consists of $n$ elements of value between 1 and $2 k+10 \sqrt{k}$, which gives us $(2 k+10 \sqrt{k})^{n}$ possible evaluations for this sequence. For every fixed route $R$ with $m$ peaks corresponding to the repeated block of lengths $p_{1}, \ldots, p_{m}$ there are at most $p_{1} p_{2} \ldots p_{m}$ sequences which can occur as $P$. Therefore the upper bound for the number of possible sequences $P$ can be obtain by determining maximum value of the product $p_{1} p_{2} \ldots p_{m}$ with $p_{1}+\ldots+p_{m}=M$. The inequality between the arithmetic and geometric means implies that the maximum is obtained when all $p_{i}$ are the same. Denote their common value by $x$. We must determine $\max \left(x^{\frac{M}{x}}\right)$. Since

$$
\left(x^{\frac{M}{x}}\right)^{\prime}=x^{\frac{M}{x}}\left(\frac{M}{x^{2}}-\frac{M \log (x)}{x^{2}}\right),
$$

we get that the maximum value is obtained with $x=e$ and equals $\approx 1.44467^{M}<1.5^{M}$. All these bounds brings us to the conclusion that the number of possible logs exceeds

$$
(2 k+10 \sqrt{k})^{n} C_{M} k^{M / 2} 2^{M / 2}(1.5)^{M} .
$$

Comparing with the number of evaluations of a sequence $\left(r_{j}\right)$ we get the inequality

$$
(10 \sqrt{k})^{M} \leq(2 k+10 \sqrt{k})^{n} C_{M} k^{M / 2} 2^{M / 2}(1.5)^{M} .
$$

Asymptotically, Catalan numbers $C_{n}$ grow as $\frac{4^{n}}{n^{3 / 2} \sqrt{\pi}}$ which implies that

$$
(10 \sqrt{k})^{M} \leq(2 k+10 \sqrt{k})^{n} \frac{4^{M}}{M \sqrt{\pi M}} k^{M / 2} 2^{M / 2}(1.5)^{M} .
$$

The right hand side is $o\left((10 \sqrt{k})^{M}\right)$ therefore for large enough $M$ the inequality can not hold. We get a contradiction, from which we conclude that for some specific choices of $r_{1}, r_{2}, \ldots$ the Algorithm stops.

The above proof can be applied in a more general setting.

Theorem 2. Let $K$ be a fixed set of $k$ positive integers. For every $n \geqslant 1$ and for any sequence of sets $L_{1}, \ldots, L_{n}$ of size at least $2 k+10 \sqrt{k}$ each, there exists a sequence $S=s_{1} \ldots s_{n}$ with $s_{i} \in L_{i}$ for all $i=1,2, \ldots, n$ which is nonrepetitive on every arithmetic progressions whose common difference is in $K$.

In the proof of Theorem 1, we focused only on the number of forbidden substructures, not their values. Given an arbitrary set of common differences we order and numerate them from 1 up to $k$. We can repeat the above reasoning with just one change - the sequence $D$ consists of elements of $K$ (but there are still $k$ of them). 


\section{A related geometric problem}

As stated in the introduction, the problem of finding sequences nonrepetitive up to mod $k$ has its origin in a geometric problem of nonrepetitive coloring of points in the plane. We can apply our proof technique to a more general question in this setting. The following problem concerning nonrepetitive colorings of discrete sets of points in $\mathbb{R}^{n}$ was considered in [9]. Let $P$ be a discrete set of points and let $L$ be a fixed set of lines in $\mathbb{R}^{n}$. A coloring of $P$ is nonrepetitive (with respect to $L$ ) if each line in $L$ is colored nonrepetitively (i.e., no sequence of consecutive points on any $l \in L$ forms a repetition). For a point $p \in P$ let $i(p)$ denote the number of lines from $L$ incident with $p$ and let $I=I(P, L)=\max \{i(p): p \in P\}$ be the maximum incidence of the configuration $(P, L)$. Using the Lovász Local Lemma it was proved in [9] that $I e^{\left(8 I^{2}+8 I-4\right) /(I-1)^{2}}$ colors are sufficient to get such a coloring. Adopting the proof of Theorem 2 we can get a better bound.

Theorem 3. Let $(P, L)$ be a configuration of points and lines in $\mathbb{R}^{n}$ with finite maximum incidence $I>2$. If $C \geqslant 2 I+10 \sqrt{I}$, then there is a nonrepetitive $C$-coloring of $P$ with respect to $L$.

Proof. The argument is pretty much the same as in the proof of Theorem 1 . We provide an algorithm for which each point is colored at random by one of $2 I+10 \sqrt{I}$ colors. Fix any linear ordering of all points in $P$. We color them in this order using Algorithm 1, where arithmetic progressions are changed into lines in $\mathbb{R}^{n}$. Similarly, for a given point $p \in P$ and every line $l \in L$ such that $p \in l$ we forbid to use colors already assigned to $I$ points preceding and following $p$ on $l$. This gives us at most $2 I$ forbidden colors for each point. Similarly to the previous proof, one can show that additional $10 \sqrt{I}$ colors suffice to get a nonrepetitive coloring of $P$ with respect to $L$. For a $\log (R, D, O, P, S)$ we take the same objects as in last case, with the exception that now $D$ keeps the information about the line for which we get a repetition (values between 1 and $I$ ), and $S$ is a sequence of numbers between 0 and $I$. From this point all calculations run similarly as before.

\section{An open problem}

We would like to conclude the paper with a problem concerning infinite sets of forbidden differences. Let $K$ be a fixed (possibly infinite) set of positive integers. A coloring of the integers is $K$-nonrepetitive if every arithmetic progression with common difference in $K$ forms a nonrepetitive sequence. Denote by $\pi(K)$ the minimum number of colors (possibly infinite) needed for a $K$-nonrepetitive coloring of $\mathbb{Z}$.

A natural question is for which sets $K$ the number $\pi(K)$ is finite. An obvious necessary condition is that the related integer distance graph (i.e., a graph on the set of vertices $\mathbb{Z}$ with two integers $a>b$ joined by an edge whenever their difference $a-b$ is in $K$ ) has finite chromatic number, denoted by $\chi(K)$. Theorem 2 shows that $\pi(K)$ is finite for finite sets $K$. More intriguing in this respect is the case of infinite sets $K$. We offer the following conjecture in the spirit of Erdős. 
Conjecture 2. $\pi(K)$ is finite for every lacunary set $K$.

A set $K=\left\{k_{1}<k_{2}<\ldots\right\}$ is lacunary if there is a real number $\delta>0$ such that

$\frac{k_{i+1}}{k_{i}}>1+\delta$ for all indices $i$. For instance the set of powers of 2 and the set of Fibonacci numbers are lacunary. It is known that for such sets the usual chromatic number $\chi(K)$ is finite (see $[13,16,17])$. However, there are non-lacunary sets with a finite chromatic number. Complete characterization of such sets is not known and, as pointed out by Ruzsa (personal communication), this problem is connected to some deep questions in additive number theory. A trivial example is the set of odd positive integers, whose chromatic number is 2. Curiously, for the nonrepetitive variant just 4 colors suffice as proved by Carpi [6], which supports even stronger supposition that perhaps $\pi(K)$ is finite if and only if $\chi(K)$ is finite.

\section{References}

[1] J-P. Allouche, J. Shallit, Automatic sequences. Theory, applications, generalizations, Cambridge University Press, Cambridge, 2003.

[2] N. Alon, J.H. Spencer, The probabilistic method, Second Edition, John Wiley \& Sons, Inc., New York, 2000.

[3] D. R. Bean, A. Ehrenfeucht, G. F. McNulty, Avoidable patterns in strings of symbols, Pacific J. Math. 85 (1979), 261-294.

[4] J. Berstel, Axel Thue's work on repetitions in words; in P. Leroux, C. Reutenauer (eds.), Séries formelles et combinatoire algébrique Publications du LaCIM,, Université du Québec a Montréal, p 65-80, 1992.

[5] J. Berstel, Axel Thue's papers on repetitions in words: a translation, Publications du LaCIM, vol 20, Université du Québec a Montréal, 1995.

[6] A. Carpi, Multidimensional unrepetitive configurations, Theoret. Comput. Sci. 56 (1988) 233241.

[7] J. Currie and J. Simpson, Non-repetitive Tilings, The Electron. J. Comb., 9 (2002), $2-8$.

[8] J. Currie, Pattern avoidance; themes and variations, Theor. Comput. Sci., 339 (2005), $7-18$.

[9] J.Grytczuk, Thue-Like Sequences and Rainbow Arithmetic Progressions, The Electr. J. Comb., 9(1) (2002), Research Paper 44, 10.

[10] J. Grytczuk, Nonrepetitive colorings of graphs - a survey, Int. J. Math. Math. Sci. (2007), Art. ID 74639, 10 pp.

[11] J. Grytczuk, Thue type problems for graphs, points, and numbers, Discrete Math. 308 (2008) 4419-4429.

[12] J. Grytczuk, J. Kozik, P. Micek, A new approach to nonrepetitive sequences (submitted). 
[13] Y. Katznelson, Chromatic numbers of Cayley graphs on $\mathbb{Z}$ and recurrence, Combinatorica 21 (2001), 211219.

[14] M. Lothaire, Combinatorics on Words, Addison-Wesley, Reading MA, 1983.

[15] R. Moser, G. Tardos, A constructive proof of the general Lovász local lemma, J. ACM, 57 (2010), Art. 11, 15.

[16] Y. Peres, W. Schlag, Two Erdős problems on lacunary sequences: Chromatic number and Diophantine approximation, Bull. London Math. Soc. 42 (2010) 295300.

[17] I. Ruzsa, Z. Tuza, M. Voigt, Distance graphs with finite chromatic number, J. Combin. Theory, Ser. B, 85 (2002), 181-187.

[18] A. Thue, Über unendliche Zeichenreichen, Norske Vid. Selsk. Skr., I Mat. Nat. Kl., Christiania, 7 (1906), 1-22. 